\title{
Centile-based Early Warning Scores derived from statistical distributions of vital signs
}

\author{
Lionel Tarassenko a , David A. Clifton ${ }^{\text {a }}$, \\ Michael R. Pinsky ${ }^{b}$, Marilyn T. Hravnak ${ }^{b}$, John R. Woods ${ }^{\text {c }}$, and Peter J. Watkinson ${ }^{\text {a,d }}$ \\ ${ }^{a}$ University of Oxford, UK \\ ${ }^{\mathrm{b}}$ University of Pittsburgh Medical Centre, US \\ ${ }^{\mathrm{c}}$ Clarian Health Partners, Indianapolis, US \\ ${ }^{\mathrm{d}}$ Oxford Radcliffe Hospitals NHS Trust, UK
}

Corresponding author:

Prof. Lionel Tarassenko

Institute of Biomedical Engineering

Old Road Campus Research Building (off Roosevelt Drive)

University of Oxford, Oxford OX3 7DQ, UK

Phone: +44 1865 617674, Fax: +44 1865617701

e-mail: lionel.tarassenko@eng.ox.ac.uk

\begin{abstract}
Aim of study: To develop an Early Warning Score (EWS) system based on the statistical properties of the vital signs in at-risk hospitalised patients.

Materials and methods: A large dataset comprising 64,622 hours of vital-sign data, acquired from 863 acutely-ill in-hospital patients using bedside monitors, was used to investigate the statistical properties of the four main vital signs. Normalised histograms and cumulative distribution functions were plotted for each of the four variables. A centile-based alerting system was modelled using the aggregated database.

Results: The means and standard deviations of our population's vital signs are very similar to those published in previous studies. When compared with EWS systems based on a future outcome, the cut-off values in our system are most different for respiratory rate and systolic blood pressure. With four-hourly observations in a 12-hour shift, about 1 in 8 at-risk patients would trigger our alerting system during the shift.

Conclusions: A centile-based EWS system will identify patients with abnormal vital signs regardless of their eventual outcome and is therefore more likely to generate an alert when presented with patients with redeemable morbidity or avoidable mortality. We are about to start a stepped-wedge clinical trial gradually introducing an electronic version of our EWS system on the trauma wards in a teaching hospital.
\end{abstract}

Abstract word-count: 211 words

Manuscript word-count: 2998 words 


\section{Introduction}

In 1997, Morgan and colleagues described the first early warning score (EWS) system, designed to alert clinicians to deteriorating patients using aggregate weighted scoring of vital signs. ${ }^{1}$ Many variations of this scheme have since been published. ${ }^{2}$ Despite evidence that physiological instability precedes critical clinical deterioration, ${ }^{3,4,5,6}$ EWS systems have not been shown to improve patient outcomes. ${ }^{2,7,8}$

Most EWS systems use weights and cut-off values for vital signs that are derived from expert opinion. ${ }^{2,9,10}$ We argue that clinical conjecture can be improved upon by statistical determination of the ranges of normality in the at-risk population, for each vital sign observation. In this paper we describe the development of a centile-based system derived from such information.

\section{Methods}

\subsection{Data collection}

A large dataset comprising 64,622 hours of vital-sign data, acquired from 863 acutely-ill inhospital patients using bedside monitors, was used to investigate the statistical properties of the four main vital signs: heart rate (HR), respiratory rate (RR), peripheral arterial oxygen saturation $\left(\mathrm{SpO}_{2}\right)$, and systolic blood pressure $(\mathrm{SysBP})$. The data came from three clinical studies in the United Kingdom (UK) and United States (US) between 2004 and 2008. ${ }^{6,11,12,13}$

The methodologies of these studies have previously been described. The CALMS1 study (Oxford, UK, OxREC No:C03.057) ${ }^{6}$ comprised a randomised controlled trial of the effects of mandated continuous physiological monitoring in both medical and surgical ward patients with a $>5 \%$ risk of in-hospital death. Patients were randomised to receive either mandated continuous 5parameter physiological monitoring (for up to 72 hours post-surgery or post acute medical admission), or to receive usual ward care. The Clarian Health study (Indianapolis, US, approved by the Clarian Methodist Hospital Institutional Review Board) ${ }^{11}$ was a prospective cohort study of the use of monitoring to detect cardio-respiratory instability in general medical/surgical patients receiving treatment on a Progressive Care Unit in a teaching hospital. The University of Pittsburgh Medical Center (UPMC) study (Pittsburgh, US, approved by the UPMC Patient 
Safety Committee) ${ }^{12}$ was a quality improvement project for a 24-bed adult surgical trauma StepDown Unit in a level 1 trauma center hospital. In Phase 1 (standard care), continuous vital-sign data were collected from bedside monitors over eight weeks. Phase 3 was an eight-week intervention phase, ${ }^{13}$ during which nursing staff utilised a clinical algorithm to respond to alerts from a data fusion system (Visensia, OBS Medical, Abingdon, UK) connected to the bedside monitors. Phase 2 was a training phase for the nursing staff.

\subsection{Statistics}

Normalised histograms (unit area under the curve) and cumulative distribution functions were plotted for each physiological variable (HR, RR, $\mathrm{SpO}_{2}$ and $\mathrm{SysBP}$ ), for each study and for the 3 datasets combined.

\subsection{Data utilisation}

A centile-based alerting system for the four variables was modelled using the aggregated database. The alerting system was constructed using the hypothesis that an EWS of 3 (which, in most systems, initiates a review of the patient) should be generated when a vital sign is below the $1^{\text {st }}$ centile or above the $99^{\text {th }}$ centile for that variable (for a double-sided distribution). We further considered that a score of 2 should correspond to the vital sign being between the $1^{\text {st }}$ and $5^{\text {th }}$ centiles or between the $95^{\text {th }}$ and $99^{\text {th }}$ centiles and that a score of 1 should correspond to the vital sign being between the $5^{\text {th }}$ and $10^{\text {th }}$ centiles or between the $90^{\text {th }}$ and $95^{\text {th }}$ centiles. $\left(\right.$ For $\mathrm{SpO}_{2}$, with a one-sided distribution starting at $100 \%$, values above the $98^{\text {th }}$ centile will give a score of 3 , values between the $90^{\text {th }}$ and $98^{\text {th }}$ centiles a score of 2 , and values between the $80^{\text {th }}$ and $90^{\text {th }}$ centiles a score of 1 ).

\subsection{Alert generation}

The number of alerts which would be generated by our proposed EWS system was investigated, assuming that alerts occur whenever a score of 3 is assigned to a single variable and a score of $\geq 4$ for the multivariate case.

To estimate the expected number of alerts generated for different frequencies of patient observation, the four vital signs in the database were evaluated using the proposed centile-based 
EWS at intervals of $N$ hours, for $N=4,8$, and 12 hours. The median of each vital sign was calculated over the 1-minute interval centred on the observation time.

\section{Results}

The patient population characteristics in each study are shown in Table 1 .

\begin{tabular}{|c|c|c|c|c|}
\hline & CALMS1 & Clarian & UPMC (P1) & UPMC(P3) \\
\hline Number of patients & 169 & 70 & 324 & 300 \\
\hline $\begin{array}{c}\text { Age } \\
\text { (mean } \pm \mathrm{sd})\end{array}$ & $71 \pm 14$ & $59 \pm 19$ & $58 \pm 20$ & $57 \pm 20$ \\
\hline $\begin{array}{c}\text { Age } \\
\text { (median } \pm \mathrm{IQR})\end{array}$ & $74 \pm 18$ & $65 \pm 23$ & $59 \pm 29$ & $58 \pm 28$ \\
\hline Sex (Male) & $57.4 \%$ & $44.3 \%$ & $58.9 \%$ & $58.7 \%$ \\
\hline Medical & $63.3 \%$ & $70.0 \%$ & $13.5 \%$ & $6 \%$ \\
\hline Surgical & $36.4 \%$ & $30.0 \%$ & $86.5 \%$ & $94 \%$ \\
\hline Mortality & $9.8 \%$ & $4.3 \%$ & $2 \%$ & $1 \%$ \\
\hline
\end{tabular}

Table 1 - Patient demographics for the CALMS1 (Oxford, UK), Clarian (Indianapolis, US) and UPMC (Pittsburgh, US) studies

\subsection{Vital sign characteristics}

The distributions for each variable (see Table 2 for means and standard deviations) were very similar in all studies; acutely-ill hospital patients in the UK and US have the same physiological characteristics. The vital sign data were aggregated together to produce an overall histogram for each variable. These histograms, computed from the 64,622 hours of data, are shown in Figure 1. The HR histogram shows small peaks at 60 and 70 beats/minute, corresponding to the common settings for patients fitted with pacemakers.

\begin{tabular}{|c|c|c|c|c|}
\hline & Heart rate & Respiratory rate & $\mathrm{SpO}_{2}$ & Systolic BP \\
\hline JR (CALMS1) & $85.0(16.8)$ & $19.8(7.3)$ & $96.1(3.8)$ & $118.5(24.5)$ \\
\hline Clarian & $84.0(18.6)$ & $19.0(5.4)$ & $95.4(3.2)$ & $129.7(23.5)$ \\
\hline UPMC (P1) & $83.1(16.9)$ & $18.5(5.4)$ & $96.0(4.4)$ & $128.1(20.6)$ \\
\hline UPMC (P3) & $82.8(17.0)$ & $18.3(5.3)$ & $96.3(3.5)$ & $127.6(20.0)$ \\
\hline Total & $\mathbf{8 4 . 2 ( 1 7 . 4 )}$ & $\mathbf{1 8 . 6 ( 5 . 3 )}$ & $\mathbf{9 6 . 0}(\mathbf{3 . 7})$ & $\mathbf{1 2 8 . 5 ( 2 1 . 4 )}$ \\
\hline
\end{tabular}

Table 2 - Vital sign means (standard deviations) from our database (CALMS1, Clarian and UPMC studies) 

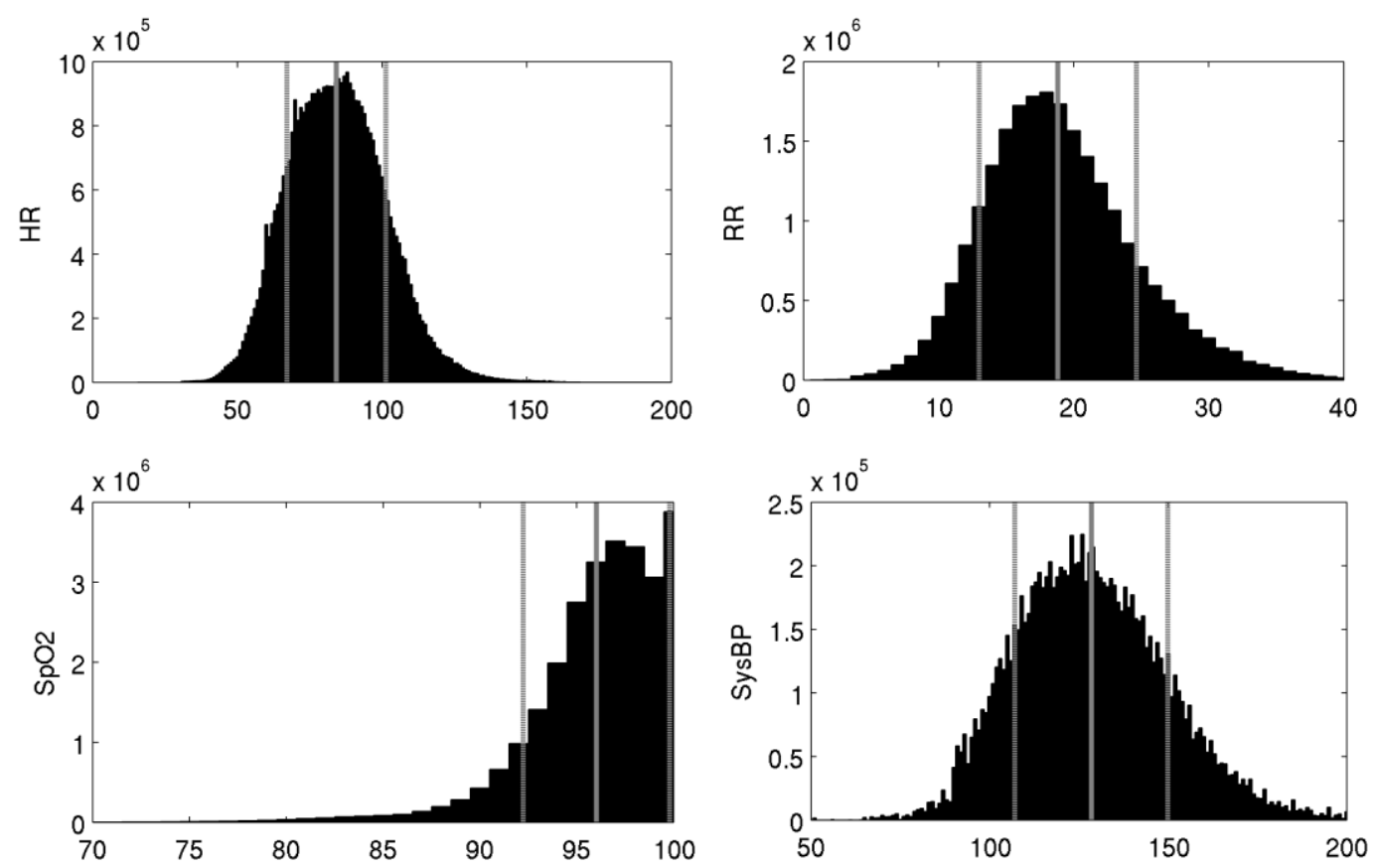

Figure 1 - Histograms for heart rate, breathing rate, arterial oxygen saturation and systolic blood pressure, computed from 64,622 hours of vital sign data acquired from 863 patients. The central vertical line indicates the mean of the data, with the two vertical lines either side corresponding to one standard deviation (except for $\mathrm{SpO} 2$, which has a one-sided distribution).

\subsection{Weightings and cut-offs estimated from vital-sign database}

Histograms are estimates of the probability density function $p(x)$ for the random variable $x$. The cumulative distribution function (cdf), $P(x)$, is the integral of $p(x)$. The cdfs for each vital sign are shown in Figure 2. The vertical lines on these plots allow the cut-off values to be determined for each vital sign. To take respiratory rate as an example, $1 \%$ of patients had a respiratory rate $\leq 7 \mathrm{breaths} / \mathrm{min}, 5 \%$ a rate $\leq 10 \mathrm{breaths} / \mathrm{min}$, and $10 \%$ a rate $\leq 13 \mathrm{breaths} / \mathrm{min}$. At the upper end, $90 \%$ of patients had a respiratory rate $\leq 26$ breaths $/ \mathrm{min}, 95 \%$ a rate $\leq 29$ breaths $/ \mathrm{min}$ and $99 \%$ a rate $\leq 34$ breaths $/ \mathrm{min}$.

The information given in Figure 2 can be converted to the tabular format used in track-andtrigger forms, as in Table 3. 

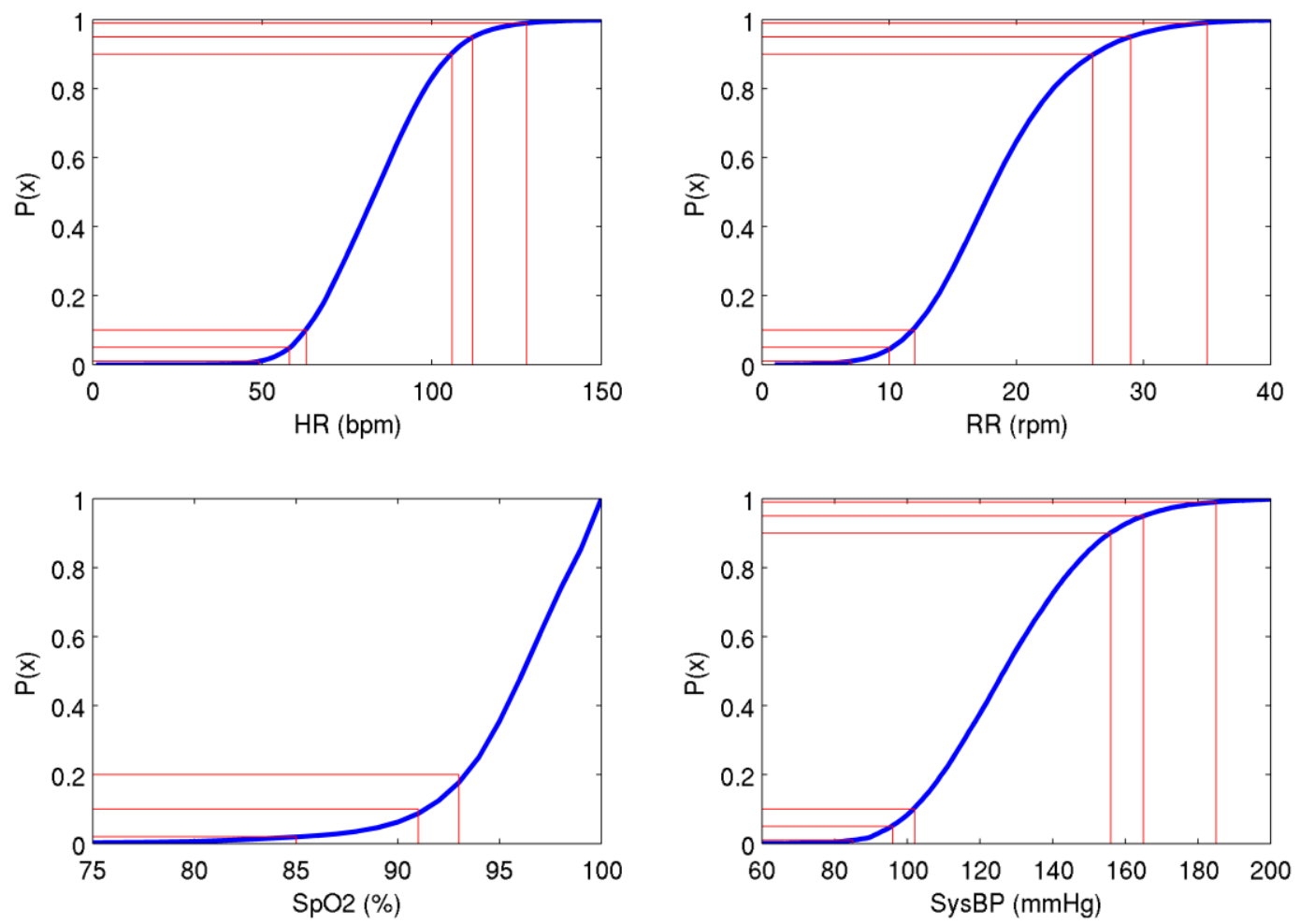

Figure 2 - Cumulative distribution function (cdf) plots for heart rate, respiratory rate, $\mathrm{SpO}_{2}$ and systolic blood pressure, computed from 64,622 hours of vital sign data acquired from 863 patients. The $[1,5,10,90,95,99]$ centiles are shown on the vertical axis and the corresponding cut-off values on the horizontal axis. For $\mathrm{SpO}_{2}$, which has a one-sided distribution, the [80, 90, 98] centiles are used instead.

\begin{tabular}{c|c|c|c|c|c|c|c}
\multicolumn{1}{c}{} & 3 & 2 & \multicolumn{1}{c}{1} & 0 & 1 & 2 & 3 \\
\hline $\mathrm{RR}$ & $\leq 7$ & $8-10$ & $11-13$ & $14-25$ & $26-28$ & $29-33$ & $\geq \mathbf{3 4}$ \\
\hline $\mathrm{HR}$ & $\leq 50$ & $51-58$ & $59-63$ & $64-104$ & $105-112$ & $113-127$ & $\geq \mathbf{1 2 8}$ \\
\hline $\mathrm{SBP}$ & $\leq 85$ & $86-96$ & $97-101$ & $102-154$ & $155-164$ & $165-184$ & $\geq 185$ \\
\hline $\mathrm{SpO}_{2}$ & $\leq 84$ & $85-90$ & $91-93$ & $\geq 94$ & & & \\
\hline
\end{tabular}

Table 3 - Range of values for each weighting for the main vital signs (RR, HR, SysBP and $\mathrm{SpO}_{2}$ ) in the proposed centile-based EWS derived from evidence base of 64,622 hours of continuously recorded vital sign data from 863 patients 


\subsection{Number of alerts}

Table 4 shows the percentage of the patient population that would generate one or more alerts within $N$ hours, when using our proposed centile-based EWS system with different frequencies of vital-sign observation. $N$ can be thought of as the time interval during which a clinical team is on duty (e.g. $N=12$ for a 12 -hour shift) and $M$ is the time interval between observations (e.g. $M$ $=4$ for four-hourly observations). $M$ is always less or equal to $N$.

\begin{tabular}{|c|c|c|c|}
\cline { 2 - 4 } \multicolumn{1}{c|}{} & \multicolumn{3}{c|}{ Shift length (duration of observations - N) } \\
\hline Observation frequency $(\mathrm{M})$ & 4 hours & 8 hours & 12 hours \\
\hline 4 hourly & $5.1 \%(4.1 \%)$ & $8.9 \%(7.1 \%)$ & $12.2 \%(9.9 \%)$ \\
\hline 8 hourly & N/A & $5.1 \%(4.1 \%)$ & $7.0 \%(5.8 \%)$ \\
\hline 12 hourly & N/A & N/A & $5.3 \%(4.4 \%)$ \\
\hline
\end{tabular}

Table 4 - Percentage of patients that generate an alert (total score of 4 or more, or single variable with a score of 3) within a period of $\mathrm{N}$ hours with centile-based EWS, if vital signs are observed every $\mathrm{M}$ hours. The figure in brackets indicates the percentage of patients generating an alert as a result of a single vital sign scoring 3 .

The percentage of patients that generate an alert decreases with decreasing frequency of observations, because each patient is being observed less often. When observations are made every four hours during a 12-hour shift, approximately $12 \%$ of the at-risk patients ( 1 in 8 ) would be expected to generate an alert during the shift.

This analysis allows us to estimate the resource required to attend to patients as a function of observation frequency. The program for performing this type of analysis with a user-defined EWS is available at http://www.robots.ox.ac.uk/ davidc/EWS. As the patients in our database were considered to be at high risk of clinical deterioration, the numbers in Table 4 may overestimate the population alerting rates for the whole spectrum of hospitalised in-patients.

\section{Discussion}

We have presented the ranges of values for the four main vital signs in at-risk hospitalised populations. We suggest that clinicians would want to be alerted when their patient strays outside the limits of normality defined by these ranges. Conversely, it seems reasonable that patients 
whose vital signs remain within the limits of normality should not score in an alerting system. Our EWS uses the $10^{\text {th }}$ and $90^{\text {th }}$ centiles to define the no-score limits. A centile-based system provides a statistical basis for identifying vital signs outside the population limits of normality.

In comparison with existing EWS systems, our cut-off values differ most for RR and SysBP. Most acutely-ill patients in hospital have elevated respiratory rates, as indicated by a mean value of 18.6 (SD 5.3) breaths/min (Table 2). In most current EWS, respiratory rates of 24 breaths/min, i.e. within one SD of the mean (broadly normal for the population), score highly. ${ }^{8,14,15,16}$ This may be because clinicians base "normal" values on those for young healthy populations, rather than older, hospitalised populations. SysBP shows the opposite behaviour. Several EWS systems do not score for hypertension $\leq 250 \mathrm{mmHg},{ }^{7,14,15}$ yet a patient with a SysBP of $190 \mathrm{mmHg}$ is in the highest centile for blood pressure and so would be reviewed in our system. Hypertension, unlike hypotension, does not appear to be associated with imminent mortality, ${ }^{14,17}$ but this does not make it a poor marker of early clinical deterioration. If a patient is chronically hypertensive, the EWS system can be adjusted (at the first alert) to take account of this. If the hypertension is caused instead by an event leading to pain or distress, it may be an earlier marker of deterioration than when cardiovascular decompensation (with hypotension) occurs.

Rather than relying solely on expert opinion, recent EWS systems ${ }^{10,14,15,18}$ (e.g. the ViEWS system $^{14}$ ) use a binary outcome (mortality/survival or admission/no admission to ICU). The cutoff values for each vital sign (measured at time T1) are set such that alerts are meant to predict the binary outcome at some later time T2 (often 24 hours after T1). The prediction accuracy is assessed by comparing the Areas-Under-the-Receiver-Operator-Characteristic (AUROC), improvements being achieved by adjusting the cut-offs for each vital sign. ${ }^{10,14}$ However, in any patient cohort used to create these EWS systems, some form of vital-sign monitoring will have been used (if only a traditional TPR chart). Many of the patients will have displayed vital sign abnormalities (identified by the chart), which led to an escalation of care at T1. Some of the patients in the "good outcome" group at T2 would have been in the "poor outcome" group but for the escalation of care triggered by the use of the chart at $\mathrm{T} 1$. 


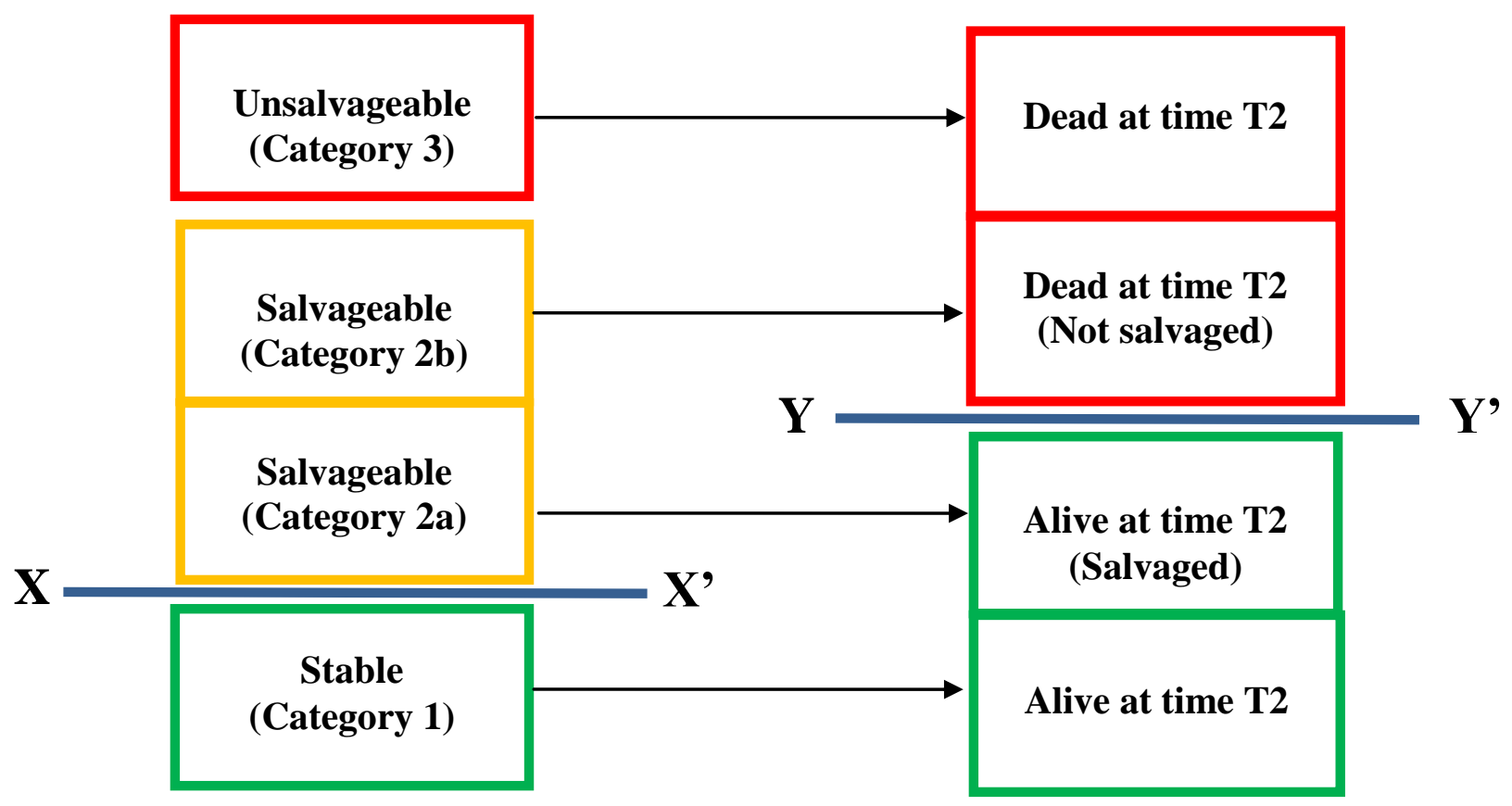

Figure 3 - Vital-sign measurements are made at time T1 when there are 3 categories of patients (stable, salvageable and unsalvageable) which are not known to the observer. There are only 2 outcomes at time T2 (dead or alive) separated by the classification boundary YY'

As shown in Figure 3, there are three categories of patients at T1, but these are not known to the observer: patient will die regardless of intervention ("unsalvalgeable"); patient will survive, even without intervention ("stable"); and patient may be "salvageable" as a result of the intervention triggered by the observation of abnormal vital signs. Ideally, AUROC optimisation would be carried out using the three categories at T1 to set the EWS threshold for alerting at its optimal value, that which corresponds to the $\mathrm{XX}$ ' boundary in Figure 3. If we could use $\mathrm{XX}$ ' to set the alerting threshold, an intervention would not be triggered for stable patients who would be fine in any case (category 1), but an intervention would be triggered for all others (categories 2 and 3 ), thereby maximising the number of salvageable patients who are salvaged. However, we cannot use $\mathrm{XX}^{\prime}$ as the threshold for alerting as the three categories on the left-hand side of Figure 3 are not known. 
For patients in the dataset retrospectively reviewed to create the outcome-based EWS system (the "training dataset"), abnormal vital-sign observations at T1 will have triggered an intervention that resulted in a proportion of the salvageable patients (category 2) being salvaged at T2 (category 2a), although not all of them were saved (category 2b). When the outcome-based EWS system is used prospectively on new patients, it will disadvantage category 2a patients (those who were previously "salvaged" following recognition by the vital sign monitoring system in use at the time the "training dataset" was acquired). This is because an EWS threshold based on the YY' boundary will not generate an alert for these patients (since these patients in the "training dataset" belonged at T2 to the alive class) and the EWS system will therefore not prompt an intervention.

In summary, we argue that it is not valid to use AUROC optimisation to set the EWS threshold for making a decision at T1 (alert/no alert) based on classification (dead/alive) at T2, since this treats the two groups of patients below the YY' boundary in the "training dataset" as the same (same classification at T2) and hence discriminates against category 2a patients. EWS systems which use outcome data at T2 to derive a predictive rule from vital-sign measurements at T1 are based on an approach originally developed for evaluating diagnostic tests. ${ }^{19}$ Such an approach is valid in the diagnostic setting as the disease (outcome) is either present or not at the time of testing.

There is a further problem associated with choosing mortality or ICU admission as the outcome. Recent UK guidelines ${ }^{20}$ recommend that EWS systems enable prompt identification of those at risk of clinical deterioration. Focusing on vital sign values which are predictive of subsequent death/ICU admission is not necessarily the best way of finding the values which identify early deterioration, not least because delays in detecting clinical deterioration may increase morbidity without causing ICU admission or death. Unfortunately, there is no obvious binary outcome for early deterioration. Events such as escalation/no escalation of care are hard to define precisely, depend on clinical judgement ${ }^{6,21}$ and only take account of recognised deteriorations, making it impossible to set the EWS threshold for alerting on the basis of an "early deterioration outcome" at $\mathrm{T} 1$. 
Our approach relies on different assumptions. Firstly, it assumes that the vital signs of normal patients (category 1) mainly lie close to the centre of their probability distributions (i.e. they are the "most normal"), those of unsalvageable patients (category 3) at the extreme edges ("most abnormal"), and those of deteriorating but salvageable patients (category 2) somewhere between. This assumption ought not to be contentious, as it is the underlying principle of early warning scores based on vital-sign measurement. The vital-sign data from category 2a patients are included in the statistical model in the same way as the data from all other patients (avoiding the problem of mis-classification as in AUROC optimisation based on outcome at T2).

In setting the cut-off values for a score of 2 at the $5^{\text {th }}$ and $95^{\text {th }}$ centiles, we assume that the $10 \%$ most abnormal patients will score a 2 or 3 for at least one vital sign. To a first approximation, these $10 \%$ most abnormal patients consist of the $3.4 \%$ patients from categories $2 \mathrm{~b}$ and 3 (given the $3.4 \%$ mortality rate in our dataset) and of the $6.6 \%$ of patients who survived but had abnormal vital signs. Most of the patients with a score of 2 for at least one vital sign must therefore belong to category $2 \mathrm{a}$, since they are highly unlikely to be the stable category 1 patients.

Unless the vital signs of category 2 a patients are under-represented in our dataset (and hence in the probability distributions), it seems likely that these patients will alert using the proposed centiles. Of course, the eventual recognition of abnormal physiology in these patients in our dataset will have led to intervention(s) and subsequent improvement in their vital signs. However, the most abnormal vital signs for these patients will have been recorded (every five seconds) in the hours preceding clinical intervention, and our dataset contains lengthy periods of vital signs gradually becoming more abnormal for patients who alerted and subsequently recovered. ${ }^{6,12,13}$

Our system currently weights low and high deviations equally. Whether this is optimal for detecting early deterioration requires further investigation. In the 24 hours preceding death, cardiac arrest or unplanned ICU admission, low respiratory and heart rates are more prevalent than high values. ${ }^{5}$ This suggests that low rates should be given more weight. However, these low 
values may simply be markers of agonal events, rather than being of extra use in detecting early deterioration.

Our study is also limited in that it is based on retrospective analysis of high-risk hospitalised patients. However, the average values for each vital sign are remarkably similar to previously published values for hospitalised populations. ${ }^{14}$ The use of continuously-recorded data from patient monitors has allowed us to estimate the distribution of physiological variables from thousands of hours of data. Although vital-sign data from patient monitors are unvalidated, the smoothness of our cumulative distribution functions suggests that artefactual measurements are relatively low in number (and randomly distributed).

Our databases did not include detailed information on urine output and conscious level, factors commonly included in EWS systems. As urine output is a continuous variable, its cut-off values could be set as we have described. Measures of conscious level, such as the Glasgow Coma Scale (GCS) or the AVPU scale, however, are not continuous, and the mapping between the 1/2/3 EWS scores and the GCS or AVPU scales may need to be non-linear. Clearly both urine output and conscious level could be added to our proposed system. Other EWS systems also include oxygen therapy in the $\mathrm{SpO}_{2}$ score. This factor was not recorded in our dataset and its use requires investigation.

Finally, an inherent weakness of all data-based EWS systems is the low numbers of data from young people used in model construction. If (as would be expected) the pattern of physiological deteriorations differs with age, younger patients may be disadvantaged as they are underrepresented in study populations. Our initial work suggests that different cut-off values for low heart rate (bradycardia) should be used for younger adults. This topic requires further work, for all vital signs.

Despite these limitations, we believe that our methodology has produced an EWS system designed to alert the clinician to abnormal physiology in the at-risk population. As our cut-off values differ from those of previous systems, our proposed system will alert the clinician to either a different population or to a similar population at a different time. 


\section{Conclusion}

Our centile-based statistical approach is in line with the original objectives of Early Warning Scores, namely the identification of abnormal physiology at the time of the test. ${ }^{1,22}$ Observations are treated as being abnormal if they lie at the extremes of the distributions of vital signs acquired from representative sets of at-risk hospitalised patients. EWS systems based on such an approach have the potential to identify patients with abnormal vital signs and are more likely to trigger when presented with patients with redeemable morbidity or avoidable mortality (salvageable patients).

Whether the use of our system will result in improved patient outcomes needs to be tested. We are starting a stepped-wedge clinical trial gradually introducing an electronic version of our system (including temperature and AVPU) on the trauma wards in a medium-sized teaching hospital, which will enable us to measure its effect on patient outcomes.

\section{Acknowledgements}

The work described in this paper was funded by the NIHR Biomedical Research Centre Programme. Dr David Clifton is supported by the Wellcome Trust and EPSRC under grant number WT 088877/Z/09/Z.

\section{References}

1. Morgan R, Williams F, Wright M. An early warning scoring system for detecting developing critical illness. Clin Intensive Care 1997;8:100.

2. Gao H, McDonnell A, Harrison DA, et al. Systematic review and evaluation of physiological track and trigger warning systems for identifying at-risk patients on the ward. Intensive Care Med 2007;33:667-679.

3. Goldhill DR, McNarry AF, Mandersloot G, McGinley A. A physiologically-based early warning score for ward patients: The association between score and outcome. Anaesthesia 2005;60:547-553.

4. Jacques T, Harrison GA, McLaws ML, Kilborn G. Signs of critical conditions and emergency responses (soccer): A model for predicting adverse events in the inpatient setting. Resuscitation 2006;69:175-183. 
5. Krause J, Smith G, Prytherch D, et al. Intensive Care S, Australian, New Zealand Intensive Care Society Clinical Trials G. A comparison of antecedents to cardiac arrests, deaths and emergency intensive care admissions in Australia and New Zealand, and the United Kingdom the Academia study. Resuscitation 2004;62:275-282.

6. Watkinson PJ, Barber VS, Price JD et al. A randomised controlled trial of the effect of continuous electronic physiological monitoring on the adverse event rate in high risk medical and surgical patients. Anaesthesia 2006;61:1031-1039.

7. Hillman KA, Chen J, Cretikos $M$ et al. Introduction of the medical emergency team (met) system: A cluster-randomised controlled trial. Lancet 2005;365:2091-2097.

8. Subbe CP, Davies RG, Williams E at al. Effect of introducing the modified early warning score on clinical outcomes, cardio-pulmonary arrests and intensive care utilisation in acute medical admissions. Anaesthesia 2003;58:797-802.

9. Cuthbertson BH, Smith GB. A warning on early-warning scores! Br J Anaesth 2007;98:704706.

10. Cuthbertson $\mathrm{BH}$, Boroujerdi $\mathrm{M}$, McKie $\mathrm{L}$ et al. Can physiological variables and early warning scoring systems allow early recognition of the deteriorating surgical patient? Crit Care Med 2007;35:402-409.

11. Woods JR, Pohlman T, Jackson J, et al. Predictive Value of an Automated Neural-NetworkBased Early Warning System to Track the Physiological Status of Hospitalized Patients. Clarian Health Partners Technical Report, February 2009.

12. Hravnak M, Edwards L, Clontz A et al. Defining the incidence of cardio-breathing instability in step-down unit patients using an electronic integrated monitoring system. Arch Intern Med 2008;168:1300-1308.

13. Hravnak M, DeVita M, Clontz A et al. Cardio-respiratory instability before and after implementing an integrated monitoring system. Crit Care Med 2011;39:65-72.

14. Prytherch DR, Smith GB, Schmidt PE, Featherstone PI. ViEWS - Towards a national early warning score for detecting adult inpatient deterioration. Resuscitation 2010; 81:932-937.

15. Duckitt RW, Buxton-Thomas R, Walker $\mathrm{J}$ et al. Worthing physiological scoring system: Derivation and validation of a physiological early-warning system for medical admissions. An observational, population-based single-centre study. Br J Anaesth 2007;98:769-774.

16. Odell M, Rechner IJ, Kapila A, Even T, Oliver D, Davies CW, et al. The effect of a critical care outreach service and an early warning scoring system on respiratory rate recording on the general wards. Resuscitation 2007;74:470-5. 
17. Hodgetts TJ, Kenward G, Vlachonikolis IG, Payne S, Castle N. The identification of risk factors for cardiac arrest and formulation of activation criteria to alert a medical emergency team. Resuscitation 2002;54:125-31.

18. Smith GB, Prytherch DR, Schmidt PE, Featherstone PI. Review and performance evaluation of aggregate 'track and trigger' systems. Resuscitation 2008; 77:170-179

19. Altman DG, Bland JM. Statistics Notes: Diagnostic tests 3: receiver operating characteristic plots. BMJ 1994;309:188.

20. National Institute for Health and Clinical Excellence (NICE) Guideline CG50 (2007); Acutely ill patients in hospital: Recognition of and response to acute illness in adults in hospital.

21. Hillman K. What is vital to measure? Anaesthesia 2006;61:1027-1028.

22. Morgan RJ, Wright MM. In defence of early warning scores. Br J Anaesth 2007; 99(5):747748. 